\title{
GMR
}

\section{RIG-I detects HIV-1 infection and mediates type I interferon response in human macrophages from patients with HIV-1- associated neurocognitive disorders}

\author{
M.Q. Wang ${ }^{2,4}$, Y.L. Huang ${ }^{1,2}$, J. Huang ${ }^{5}$, J.L. Zheng ${ }^{1,2,3}$ and G.X. Qian ${ }^{4}$ \\ ${ }^{1}$ Laboratory of Neuroimmunology and Regenerative Therapy, \\ University of Nebraska Medical Center, Omaha, NE, USA \\ ${ }^{2}$ Department of Pharmacology and Experimental Neuroscience, \\ University of Nebraska Medical Center, Omaha, NE, USA \\ ${ }^{3}$ Department of Pathology and Microbiology, University of Nebraska Medical Center, \\ Omaha, NE, USA \\ ${ }^{4}$ Department of Biochemistry and Molecular Biology, ShanghaiJiao Tong University \\ School of Medicine, Shanghai, China \\ ${ }^{5}$ Beijing Institutes of Life Science, Chinese Academy of Sciences, Beijing, China \\ Corresponding author: G.X. Qian \\ E-mail: qinxianju@126.com \\ Genet. Mol. Res. 14 (4): 13799-13811 (2015) \\ Received April 30, 2015 \\ Accepted August 11, 2015 \\ Published October 28, 2015 \\ DOI http://dx.doi.org/10.4238/2015.October.28.42
}

\begin{abstract}
The aim of this study was to explore the precise role of retinoic acid-inducible gene-I (RIG-I) signaling in human immunodeficiency virus type 1 (HIV-1)-infected macrophages from patients with HIV-1associated neurocognitive disorders (HAND). Postmortem brain tissues were collected from patients with HIV-1-associated dementia and were compared to samples collected from HIV serum-positive patients without dementia and HIV serum-negative patients. A human monocyte-derived macrophage (MDM) primary culture system was established to evaluate the expression of RIG-I in these samples. Knockdown of RIG-I pathways
\end{abstract}


genes was employed and STAT1 expression and phosphorylation levels were examined to explore the molecular mechanisms of HAND. The expression of RIG-I in postmortem brain tissue from HAND patients was significantly higher than in patients who were HIV serum-positive without dementia or HIV serum-negative. Moreover, we demonstrated that HIV-1 infection could result in a significant increase in the level of RIG-I in human MDMs. Moreover, a correlation was found between the increase in RIG-I expression and STAT1 expression and phosphorylation. Accordingly, knockdown of RIG-I decreased the phosphorylation of STAT1 and downregulated interferon-related genes. These observations highlight the importance of RIG-I signaling in anti-HIV innate immunity in macrophages, which may be beneficial for the treatment of HIV and aid in the understanding of the neuropathogenesis of HAND.

Key words: Retinoic acid-inducible gene-I; Monocyte-derived macrophage; Human immunodeficiency virus type I;

HIV-1 associated neurocognitive disorders

\section{INTRODUCTION}

Macrophages play an important role not only in anti- human immunodeficiency virus (HIV) innate immunity of the host but also act as a target of HIV (Wang et al., 2013). Macrophages participate in the pathogenesis of HIV infection, which is highlighted by their role in viral transmission, persistence and virus dissemination (Carter and Ehrlich, 2008). Moreover, macrophages act as a virus reservoir and contribute to viral latency. They also have the ability to resist the cytopathic effects of HIV and can support long-term virus replication and production (Verani et al., 2005; Perno et al., 2006). In the setting of HIV-associated neurocognitive disorders (HAND), the invasion of HIV type 1 (HIV-1) in the central nervous system (CNS) could lead to chronic brain inflammation and progressive neurological impairment (Huang et al., 2011). As critical cellular components in the pathogenesis of HAND, macrophages are the main target of HIV infection in the brain. However, the detailed molecular mechanism on the regulation of brain macrophages during the HIV infection process has not yet been explored.

Innate immunity is the first line of HIV infection defense and viral infection could lead to the induction of the interferon (IFN)-dependent antiviral innate immune response (Katze et al., 2002; Borrow et al., 2010). Pattern recognition receptors (PRRs), including Toll-like receptors (TLR) and retinoic acid-inducible gene-I (RIG-I)-like receptor (RLRs), are major molecules involved in IFN-dependent antiviral innate immunity (Kawai and Akira, 2008). Of them, RIG-I can detect viral genomic RNA during negative-strand RNA virus infection and trigger a type I IFN-mediated immune response (Fujita et al., 2007; Rehwinkel et al., 2010). Recent studies have shown that activation of the RIG-I signaling pathway are also involved in the innate immune response during the HIV viral infection (Solis et al., 2011; Berg et al., 2012). However, the precise role of RIG-I signaling in brain macrophages from HAND patients remains unknown.

In this study, we investigated the RIG-I signaling components in postmortem brain tissue from HIV-1-associated dementia patients and explored related molecular events by using human monocyte-derived macrophage (MDM) and microglia primary culture systems. Our results demonstrate that RIG-I signaling plays an important role in macrophage anti-HIV innate immunity, which may be beneficial for the treatment of HIV and aid in the understanding of the neuropathogenesis of HAND. 


\section{MATERIAL AND METHODS}

\section{Isolation and culture of human MDMs}

Human monocytes were recovered from primary blood mononuclear cells (PBMCs) of donors positive for HIV-1 or HIV-2, and hepatitis B virus-seronegative donors after leukapheresis and purified by countercurrent centrifugal elutriation (Gendelman et al., 1988). PBMCs were seeded at a density of $1.0 \times 10^{6}$ cells/well in 24-well plates, and primary MDMs were generated by culturing adherent cells in Dulbecco's modified Eagle medium (DMEM) (GIBCO Invitrogen Corp, Carlsbad, CA, USA) with 10\% heat-inactivated pooled human serum (Cambrex Bio Science, Walkersville, MD, USA), $50 \mathrm{mg} / \mathrm{mL}$ gentamicin, $10 \mathrm{mg} / \mathrm{mL}$ ciprofloxacin (Sigma-Aldrich, St. Louis, IL, USA), and $1000 \mathrm{U} / \mathrm{mL}$ highly purified recombinant human macrophage colony stimulating factor (M-CSF) (a gift from the Wyeth Institute, Cambridge, MA, USA). Written informed consent was provided by all participants. All studies of human subjects were performed in full compliance with the University of Nebraska Medical Center and National Institutes of Health (NIH) ethical guidelines.

\section{Infection of MDM}

HIV-1 ${ }_{A D A}$ was isolated from the PBMCs from a HIV-1-infected patient with Kaposi's sarcoma as previously described (Gendelman et al., 1988). The virus was used at a multiplicity of infection (MOI) of 0.1 virus/target cell. Clade B primary viruses (D02-2562 BG, A00-086 CPx, D02-2562 Sp, and G0048 CPx) were isolated from the spleen (Sp), basal ganglia (BG), or choroid plexus (CPx) in the trigone of the lateral ventricle of infected patients with HIV encephalitis (HIVE) (Burkala et al., 2005). Clade C primary isolates (2873M, 2669M, 1084M, and 1157I) were isolated from PBMCs from a HIV-1-infected mother and infant pair in Africa (Zhang et al., 2005; Zhang et al., 2006). After human MDMs were incubated with the virus overnight, the medium was replaced with MDM culture medium and half the media was replaced every other day.

\section{Measurements of HIV reverse transcriptase (RT) activity}

HIV-1 reverse transcriptase (RT) activity was determined in triplicate samples of cell culture supernatants (Koenig et al., 1986; Zhang et al., 2006). For this assay, $10 \mathrm{~mL}$ of supernatant from macrophages (uninfected or infected with HIV) was added to a reaction mixture containing $0.05 \%$ Nonidet P-40, $10 \mathrm{mg} / \mathrm{mL}$ poly(A), $0.25 \mathrm{mg} / \mathrm{mL}$ oligo(dT), $5 \mathrm{mM} \mathrm{DTT,} 150 \mathrm{mM} \mathrm{KCl,} 15 \mathrm{mM} \mathrm{MgCl}$, and $\left.{ }^{3} \mathrm{H}\right]$ TTP. This was incubated for $24 \mathrm{~h}$ at $37^{\circ} \mathrm{C}$. Radiolabeled nucleotides were precipitated with cold $10 \%$ trichloroacetic acid on paper filters in an automatic cell harvester and washed with $95 \%$ ethanol. Radioactivity was estimated by liquid scintillation spectroscopy (Koenig et al., 1986).

\section{RNA extraction and TaqMan real-time RT-Polymerase Chain Reaction (PCR)}

Total RNA was isolated with TRIzol Reagent (Invitrogen) and RNeasy Mini Kit (QIAGEN Inc., Valencia, CA, USA) according to the manufacturer protocol. TaqMan primers used for real-time RT-PCR include human IFN-a2 (Cat. No. Hs00265051_s1), IFN-a1 (Cat. No. Hs01077958_s1), RIG-I (Cat. No. 4331182), melanoma differentiation-associated protein 5 (MDA5; Cat. No. 4331182), and human GAPDH (Cat. No. 4310884E) were purchased from Applied Biosystems, Inc. (Foster City, CA, USA). Real-time RT-PCR was carried out using 
the one-step quantitative TaqMan real-time PCR system (Applied Biosystems Inc.). Relative IFN-a2, IFN-a1, RIG-I, and MDA5 mRNA levels were determined, standardized to a GAPDH internal control, and normalized to uninfected cells using the comparative DDCT method. All primers used in the study were tested for amplification efficiencies and the results were similar to the previous study (Huang et al., 2009).

\section{Western blot analysis}

The brain tissues used in the study were acquired from the Manhattan HIV Brain Bank (kindly provided by Dr. Susan Morgello) and the Center for Neurovirology and Neurodegenerative Disorders Brain Bank (University of Nebraska Medical Center, Omaha, NE, USA). Brain tissues were cut into $0.5 \mathrm{~g}$ samples and homogenized by a Microson ultrasonic cell disruptor (Huang et al., 2011). Cell lysates from MDM and brain tissues were prepared with M-PER mammalian protein extraction buffer (Pierce) (Rockford, IL, USA) and protein concentration was determined using the BCA protein assay kit (Pierce). Protein (30 mg) was electrophoresed on pre-cast $10 \%$ SDS-PAGE and then transferred to an Immuno-Blot PVDF membrane (Bio-Rad, Hercules, CA, USA). Primary antibodies specific for phosphorylated STAT1(Y701), myeloid differentiation primary response gene 88 (MYD88), and RIG-I were obtained from Cell Signaling Technology (Danvers, MA, USA). An antibody specific for HIV-1 p24 was purchased from DAKO (Carpinteria, CA, USA). Anti-b-actin (Sigma-Aldrich) was used as a loading control. Membranes were treated overnight with primary antibody at $4^{\circ} \mathrm{C}$ followed by a secondary horseradish peroxidase-linked anti-rabbit (Cell Signaling Technology) or antimouse (Cell Signaling Technology) antibody for 1 hour at room temperature. Antigen-antibody complexes were visualized by enhanced chemiluminescence (Amersham Biosciences, Piscataway, NJ, USA) and captured with CL-X Posure ${ }^{\mathrm{TM}}$ Film (Pierce). For data quantification the films were scanned with a Canon 9950F scanner; the acquired images were then analyzed using Image J (NIH; http://rsb.info.nih.gov/nih-image/) (Huang et al., 2009).

\section{siRNA knockdown of RIG-I pathway genes}

MDMs $\left(1 \times 10^{6}\right.$ cells/well in a 24 -well plate) were transfected with pre-designed siRNA duplexes for RIG-I (Cat. No. 4392420), MyD88 (Cat. No. 4390824), MDA5 (Cat. No. 4392420) or a nonspecific control siRNA from Ambion Inc. (Austin, TX, USA) using silMPORTER (Upstate Cell Signaling Solutions, Charlottesville, VA, USA) according to the manufacturer protocol. Two rounds of transfection were performed 48 hours apart, followed by culture in DMEM containing $1 \%$ FBS. Six hours after the second transfection, the cells were either mock treated or were infected with HIV-1virus. Knockdown was confirmed by comparing to mock-infected samples (Brown et al., 2012).

\section{Statistical Analysis}

Data were expressed as mean \pm standard deviation (SD) unless otherwise specified. Multiple comparisons were performed by one way ANOVA followed by Tukey's test for paired observation. Spearman's correlation was employed to determine the association between variables and $\mathrm{P}<0.05$ was considered statistically significant. To account for any donor-specific differences, all experiments were performed with at least three donors. Assays were performed at least three times in triplicate. 


\section{RESULTS}

\section{RIG-I and MDA5 are elevated in HIVE brain tissue}

To investigate if RIG-I is involved in the pathogenesis of HAND, we evaluated RIG-I levels in the frontal cortex derived from HIV-1-infected patients, including 4 HIV-1 serum-positive individuals with dementia and 6 HIV-1 serum-positive individuals without detectable neuropathological changes (see (Huang et al., 2011) for more detail). Seven HIV serum-negative patients were employed as a negative control. RIG-I protein expression (Figure 1A, B; P < 0.01) and mRNA levels (Figure 1C; $P<0.05)$ were significantly increased in brain tissue from patients with HIV-1-associated dementia when compared with brain tissue from HIV serum-negative controls $(P<0.01)$ (Figure 1A, B). MDA5 mRNA levels were also significantly increased in brain tissue from patients with HIV-1associated dementia when compared with brain tissue from HIV-1-infected individuals without dementia or HIV serum-negative controls $(P<0.05)$ (Figure 1D).

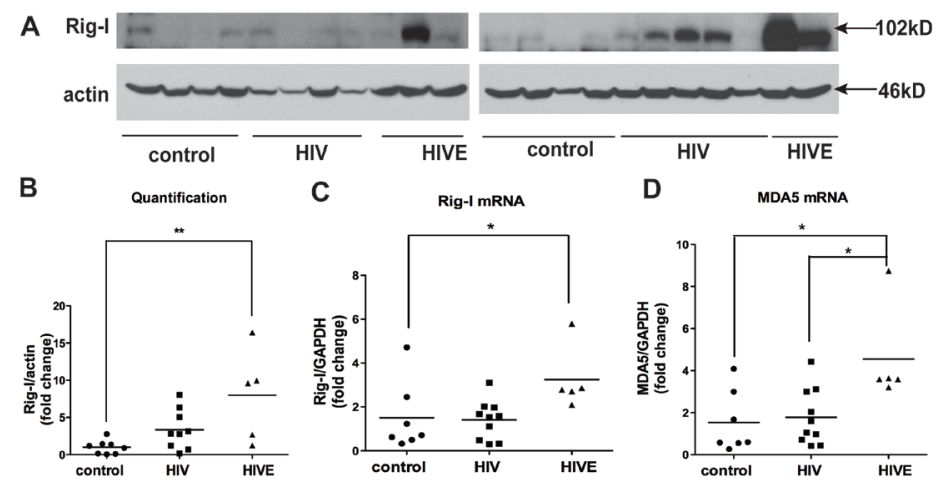

Figure 1. RIG-I is elevated in HIVE brain tissue. A. Postmortem brain tissue collected from HIV-1-associated dementia patients (HIVE), HIV serum-positive patients without dementia (HIV), and HIV serum-negative (control) patients were lysed and subjected to Western blotting for RIG-I detection. $\beta$-actin levels were used as loading controls. B. Levels of RIG-I were normalized as a ratio to $\beta$-actin and shown as fold change relative to the average of HIV serum-negative controls. ${ }^{*} \mathrm{P}<0.05$, ${ }^{* * *} \mathrm{P}<0.001$ compared with HAND patients. RIG-I expression (C) and MDA5 (D) expression in brain tissue were determined by real-time RT-PCR. Data were normalized to GAPDH and presented as fold change compared to mean value of HIV serum-negative control. ${ }^{*} \mathrm{P}<0.05$ compared with HIV serum-negative patients.

\section{RIG-I and MDA5 expression levels are correlated with the activation of type I IFN response}

To further investigate the relationship between RIG-I and type I IFN response, we analyzed RIG-I, MDA5, STAT1, IFN-a and IFN-b mRNA levels from post-mortem brain tissues collected from HAND patients, HIV serum-positive patients without dementia, and HIV serum negative individuals. We have previously reported that both protein and mRNA levels of STAT1 are significantly increased in brain tissues from HAND patients compared to HIV serum negative individuals (Huang et al., 2011; Zhao et al., 2012). Interestingly, both the protein ( $<<0.0001$; Figure $2 A)$ and mRNA ( $P=0.0117$; Figure $2 B$ ) levels of RIG-I highly correlated with those of STAT1 (P < 0.0001 ) (Figure 2A), indicating that the regulation of RIG-I may act as an immune response to HIV1 infection of the CNS. Furthermore, significant correlations were found between RIG-I and IFN-a 
$(P=0.0018)$ and IFN-b $(P=0.0107)$ (Figure $2 C$ and $D)$, indicating that RIG-I may be involved in the activation of the IFN response in HIV-1 serum-positive patients. More importantly, the MDA5 levels in these brain tissues significantly correlated with the levels of IFN-a $(P=0.0058)$ and STAT1 $(P=$ 0.04 ) (Figure 2E, F). These results indicate RIG-I and MDA5 play important roles in inducing type I IFN response in HIV dementia patients.

A

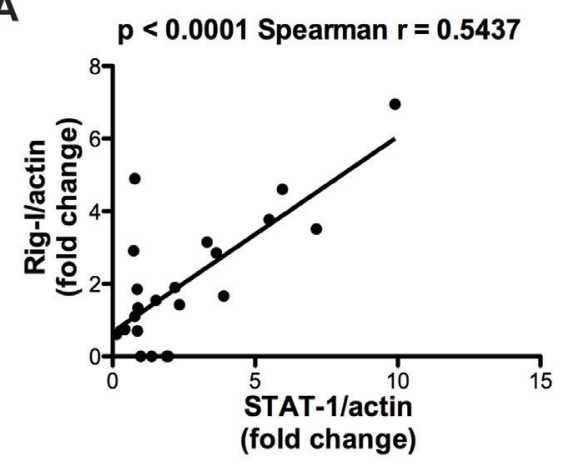

C

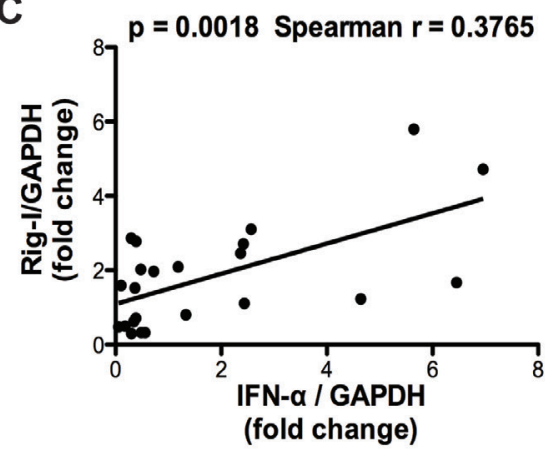

$\mathbf{E}$

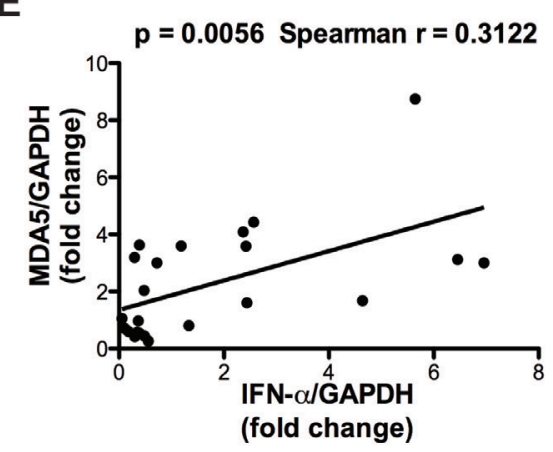

B

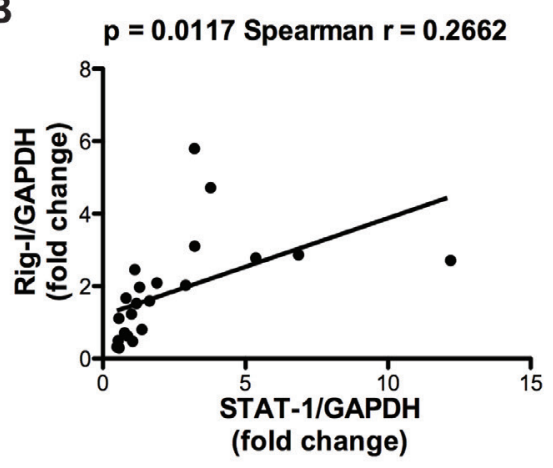

D

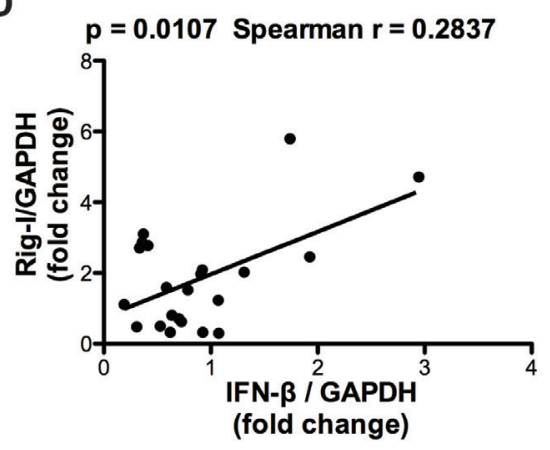

$\mathbf{F}$

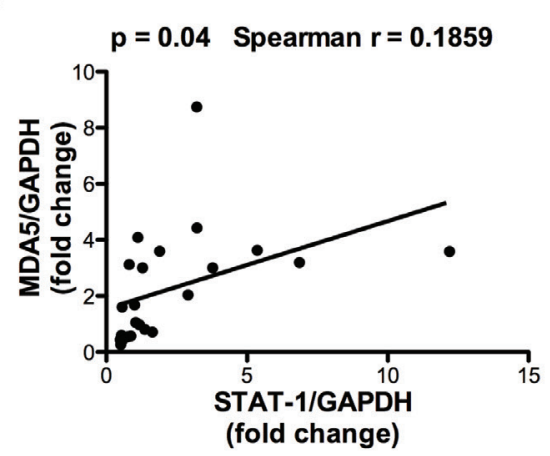

Figure 2. RIG-I and MDA5 expression correlate to the activation of type I IFN response. Correlation of RIG-I and STAT1 protein levels (A). Correlation of RIG-1 mRNA level with: STAT1 mRNA level (B), IFN- $\alpha$ mRNA level (C), and IFN- $\beta$ mRNA level (D). Correlation of MDA5 mRNA level with IFN- $\alpha$ mRNA level (E) and STAT1 mRNA level (F). All correlations were determined by Spearman's correlation. 


\section{HIV-1 infection of human MGMs induce RIG-I expression and STAT1 activation}

RIG-I is known to be involved in the activation of innate immune response in HAND patients. Since macrophages and microglia are primary sensors in the brain in HAND patients (Raivich and Banati, 2004). We next investigated whether RIG-I and MDA5 play roles in HIV-1infected human macrophages in vitro. We used macrophage-tropic HIV-1 strains HIV $_{A D A}$, clade B and clade $\mathrm{C}$ to infect human MDM. After 7, 14 and 21 days of infection, culture supernatants were collected and the efficacy of HIV-1 viral infection was determined by the HIV-1 RT activity assay. In HIV-1-infected MDM, the HIV-1 RT activity continued to increase from day 7 to day 21 (Figure 3A). We then examined the expression of RIG-I in MDM with or without HIV-1 infection. HIV-1 ${ }_{\text {ADA }}$ and clade B induced expression of RIG-I and this effect lasted for 21 days (Figure 3B, D). Since STAT1/ STAT2 signaling molecules can be activated by type I IFNs, we determined the phosphorylation level of STAT1, which is an indicator for STAT1 activation, in infected MDMs. Expression of STAT1 and STAT1 phosphorylation could be detected 7 days after HIV-1 infection, particularly after HIV ADA $_{\text {. }}$ and clade B infection, and the phosphorylation of STAT1 persisted until day 21 (Figure 3B, C). Interestingly, a significant correlation was found between RIG-I and the ratio of phosphorylated STAT1 to total STAT1 (Figure 3E). This strongly suggests that RIG-I is involved in HIV-1-induced type I IFN response in human MDMs.

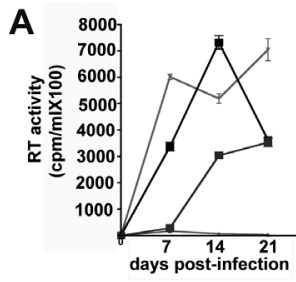

C

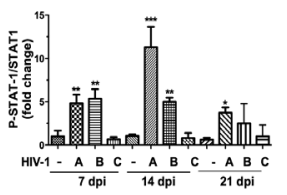

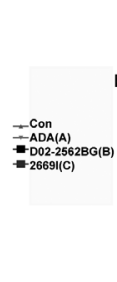

D

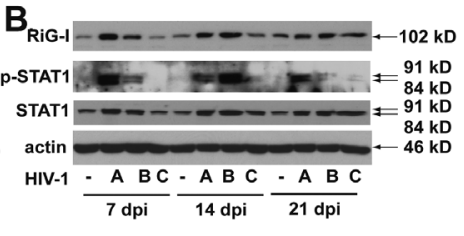

E

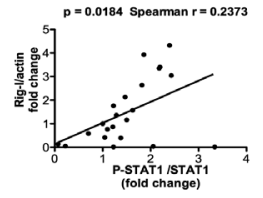

Figure 3. HIV-1 infection of human MDMs induce RIG-I expression and STAT1 activation. MDMs were infected with HIV-1ADA or HIV-1 clade B or HIV-1 clade C primary isolates (labelled as A, B, and C, respectively). Cell lysates and RNA were collected at 7, 14 and 21 days after infection. (A) HIV-1 replication was determined by RT activity assay. (B) RIG-I, phosphorylated STAT1, and total STAT1 were detected by western blot. $\beta$-actin was used as a loading control. Levels of RIG-I (C) were normalized as a ratio to $\beta$-actin and phosphorylated STAT1 (D) was normalized as a ratio to total STAT1 and shown as fold change relative to control. Results are shown as mean \pm SD in three independent experiments with three different donors. (E) Correlation of RIG-I with ratio of phosphorylated STAT1 to total STAT1 was determined by Spearman's correlation. The data are representative of three independent experiments. ${ }^{*}<0.05$ and ${ }^{* *} \mathrm{P}<0.01$ in comparison to control.

\section{Increased expression of IFN- $\alpha$ and IFN- $\beta$ expression in human MDMs after infection with HIV-1}

To determine the effect of HIV-1 infection on mRNA levels of IFNs in MDM, we used realtime RT-PCR. The expression of IFN- $\alpha$ and IFN- $\beta$ continued to increase as the infection with HIV-1 progressed, particularly with $\mathrm{HIV}-1_{\mathrm{ADA}}$ and clade B (Figure $\left.4 \mathrm{~A}, \mathrm{~B}\right)$. The mRNA levels of IFN- $\alpha$ were 
significantly higher on days 7,14 and 21 as compared to an uninfected control (Figure 4A). The IFN- $\alpha$ mRNA expression was 1.5-fold and 2.1-fold higher in HIV $_{\mathrm{ADA}}$-infected MDMs as compared to the uninfected control at days 7 and 14, respectively. For HIV-1 clade B infection, IFN- $\alpha$ mRNA was not significantly different at 7 days post-infection but was significantly higher on days 14 and 21 compared to the uninfected control. IFN- $\alpha$ mRNA levels were 4.3-fold and 5.6-fold higher in HIV clade B-infected MDMs as compared to the uninfected control on days 14 and 21, respectively. IFN- $\alpha$ mRNA levels were $80 \%$ higher in HIV clade C-infected MDMs compared to the uninfected control (Figure 4A). We obtained similar results for expression of IFN- $\beta$ and IFN- $\alpha$ in the infected MDMs. MDA5 mRNA levels were 3.8-fold, 4.4-fold and 1.8-fold higher in HIV $_{\text {ADA }}$-infected MDMs compared to the uninfected control at days 7, 14 and 21, respectively. MDA5 mRNA levels were 1.1-fold, 7.4-fold and7.5-fold higher in HIV clade B-infected MDMs compared to the uninfected control at days 7, 14 and 21, respectively. MDA5 mRNA levels were 2.2-fold higher in HIV clade C-infected to the uninfected control at days 7, 14 and 21, respectively (Figure 4C). The results indicate HIV-1 infection, including HIV-1 ${ }_{\mathrm{ADA}}$, clade $\mathrm{B}$ and clade $\mathrm{C}$, can induce MDA5 expression and activate type I IFN responses. Furthermore, a significant correlation was found between the expression of MDA5 and IFN- $\alpha(P=0.0002)$ (Figure 4D), RIG-I and IFN- $\alpha(P<0.0001)$ (Figure $4 \mathrm{E})$, and RIG-I and IFN-b $(\mathrm{P}<0.0001)$ (Figure 4F). Taken together, these data indicate that HIV-1 infection can induce RIG-I and MDA5 expression and type I IFN responses.

A

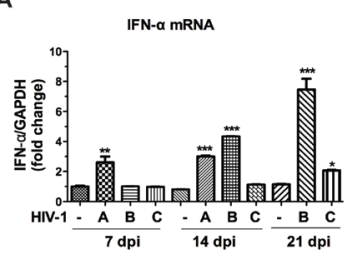

C

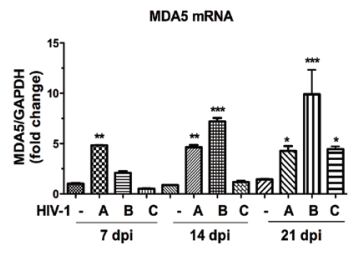

E

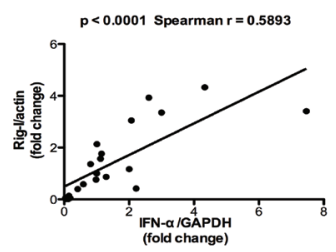

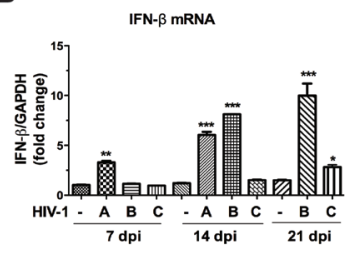

D

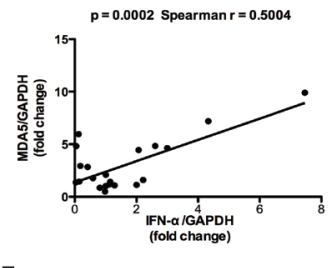

$\mathbf{F}$

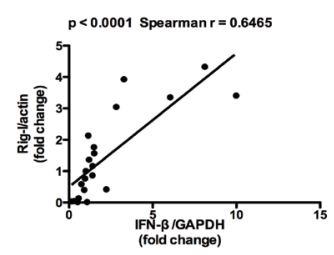

Figure 4. Increased expression of IFN- $\alpha$ and IFN- $\beta$ in human MDMs when infected with HIV-1. MDMs were infected with HIV-1ADA, HIV-1 clade B or HIV-1 clade C (labelled as A, B, and C, respectively). Cell lysates and RNA were collected 7, 14 and 21 days after infection. Real-time RT-PCR was used to detect the expression of IFN- $\alpha$ (A), IFN- $\beta$ (B) and MDA5 (C). Results were normalized to GAPDH and shown as the fold change over HIV-1 negative control. (D) Correlation of IFN- $\alpha$ with MDA5 was determined by Spearman's correlation. Correlation of IFN- $\alpha / G A P D H$ (E) or IFN- $\beta$ /GAPDH (F) with RIG-I/ $\beta$-actin was determined by Spearman's correlation. The data are representative of three independent experiments. ${ }^{*} \mathrm{P}<0.05$, ${ }^{* *} \mathrm{P}<0.01$, and ${ }^{* *} \mathrm{P}<0.001$ when compared to control. 


\section{5' ppp-dsRNA activate RIG-I and inhibit HIV-1 replication in MDMs}

$5^{\prime}$ triphosphate RNA (5' ppp-dsRNA) is a synthetic ligand for RIG-I (Marq et al., 2011) and is obtained by hybridization of non-triphosphatase single-stranded 19-mer phosphodiester RNA with its complementary strand (also non-triphosphatase). 5' ppp-dsRNA ligation significantly upregulated RIG-I expression when compared to a 5' ppp-dsRNA control (Figure 5A, B). 5' pppdsRNA also upregulated total STAT1 levels and phosphorylated STAT1. 5' ppp-dsRNA treatment appeared to inhibit HIV-1 infection by induction of STAT1 phosphorylation in MDMs (Figure 5A, C). Next, we determined HIV p24 levels to evaluate the replication of HIV-1 in MDMs. 5' ppp-dsRNA induced RIG-I activation and inhibited HIV-1 replication by $30 \%$ compared to the $5^{\prime}$ ppp-dsRNA control group (Figure 5A, D). The HIV-1 RT activity was reduced by 55\% in the 5' ppp-dsRNAtreated group compared to the control group (Figure 5F). 5' ppp-dsRNA treatment induced IFN-b expression and enhanced HIV-1-induced type I IFN responses (Figure 5E). These results indicate that 5' ppp-dsRNA induces RIG-I expression and inhibits HIV-1 replication in MDMs.

A

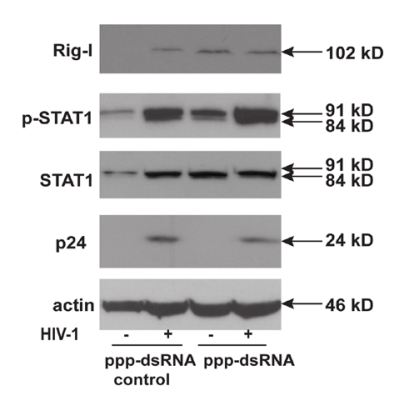

B

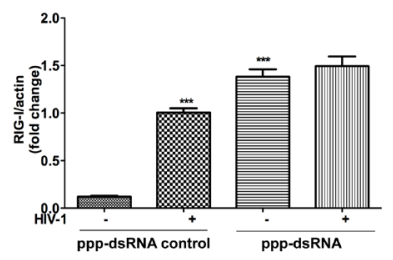

C

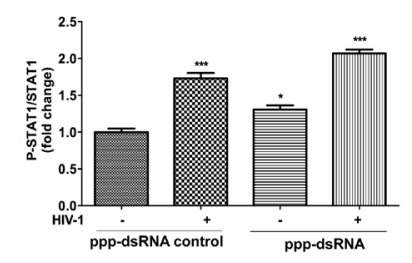

E

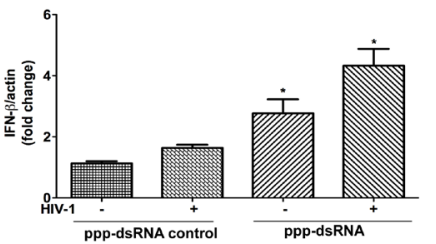

D

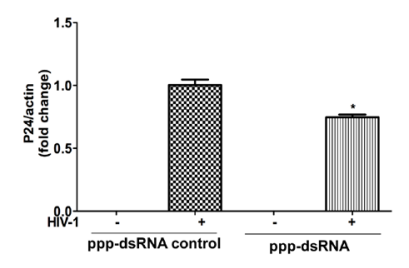

$\mathrm{F}$

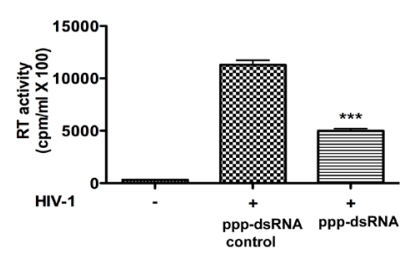

Figure 5. 5' ppp-dsRNA activates RIG-I and inhibits HIV-1 replication in MDMs. Human MDMs were transfected with $100 \mathrm{ng} / \mathrm{mL} 5$ ' ppp-dsRNA control (ppp-dsRNA control) or $100 \mathrm{ng} / \mathrm{mL}$ 5' ppp-dsRNA (ppp-dsRNA) 24 hours after HIV-1 infection. 96 hours after HIV-1 infection, protein, RNA and supernatants were collected from MDMs. (A) Phosphorylated STAT1 (P-STAT1), STAT1, RIG-I, MyD88 and P24 were detected by western blot. $\beta$-actin was used as a loading control. Levels of RIG-I (B), ratio of phosphorylated STAT1 to total STAT1 (C), and HIV p24 (D) were normalized to $\beta$-actin and shown as fold change compared to uninfected control. (E) Real-time RT-PCR was used to detect levels of IFN- $\beta$. (F) Supernatants were tested for HIV-1 RT activity. The data are representative of two independent experiments from two different donors. ${ }^{*} \mathrm{P}<0.05,{ }^{* *} \mathrm{P}<0.01$ and ${ }^{* * *} \mathrm{P}<0.001$ when compared to control. 


\section{RIG-I knockdown inhibits activation of STAT1 and enhances HIV-1 replication in HIV-1-infected MDMs}

TLRs and RIG-I-like receptors are the primary pattern recognition receptors (PRRs) that are involved in sensing RNA viruses and triggering the host IFN response. MyD88 is essential for the induction of inflammatory cytokines triggered by TLRs (Takeuchi and Akira, 2010). Accordingly, we investigated whether TLRs or a TLR pathway was responsible for sensing HIV-1 infection and subsequent induction of type I IFN. For this purpose, we studied the effect of siRNA-mediated knockdown of RIG-I, MyD88, and MDA5 expression in HIV-1-induced activation of type I IFN in MDMs. Human MDMs were transfected with siRNA against RIG-I, MyD88, MDA5 or non-targeting control siRNA. After 24h, cells were infected with HIV-1 for 3 days, followed by RNA and protein analysis. The efficacy of gene silencing at the protein level was confirmed by Western blot (Figure 6A, C, D) and mRNA levels were confirmed by real-time RT-PCR (Figure 6B). The levels of RIG-I, MyD88 and MDA5 following siRNA delivery in HIV-1-infected MDMs were 26,33 , and $27 \%$ of their levels in nonspecific siRNA-transfected HIV-infected MDMs, respectively (Figure 6A, B, C, D, E). A substantial impairment of STAT1 phosphorylation was found in MDMs transfected with siRNA for RIG-I (Figure 6A, E). In cells transfected with control siRNA, HIV-1 infection resulted in induction of RIG-I protein expression and increased STAT1 phosphorylation as expected. At the same time, HIV-1 replication was enhanced in MDMs transfected with siRNA for RIG-I compared to the control siRNA group (Figure 6A, F). These data indicate that RIG-I can detect HIV-1 infection and induce a type I IFN response in MDMs.
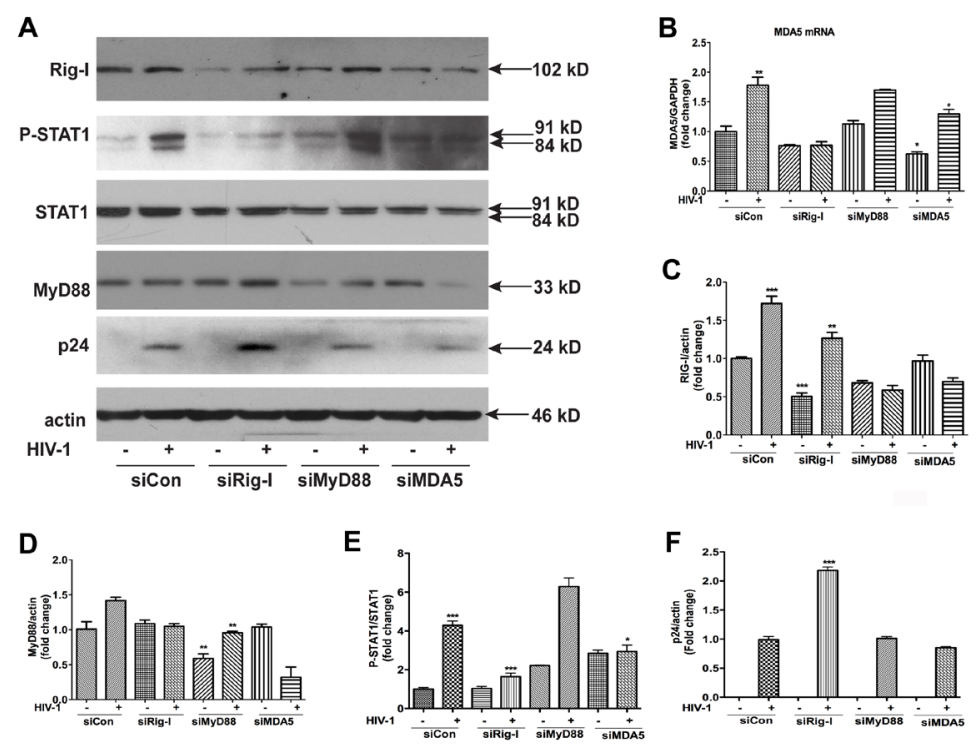

Figure 6. RIG-I silencing inhibits activation of STAT1 and enhances HIV-1 replication in HIV-1-infected MDMs. Human MDMs were transfected with RIG-I (siRIG-I), MyD88 (siMyD88), MDA5 (siMDA5) or control siRNA (siCon) 24 hours before HIV-1 infection. Seventy-two hours after HIV-1 infection, protein and RNA were collected. (A) Phosphorylated STAT1 (P-STAT1), STAT1, RIG-I, MyD88 and P24 were detected by western blot. $\beta$-actin was used as a loading control. (B) Real-time RT-PCR was used to detect MDA5 mRNA levels compared to GAPDH. Protein levels of RIG-I (C), MyD88 (D), and HIV p24 (F) were normalized to $\beta$-actin and shown as fold change compared to uninfected control. The ratio of phosphorylated STAT1 to total STAT1 is shown as fold change in (E). 


\section{DISCUSSION}

The innate immune response to HIV infection in the CNS is critical for controlling viral replication during acute infection and preserving neurological function within the immune-privileged site of the brain (Witwer et al., 2009). RIG-I represents a type of PRR and plays a crucial role in host innate immunity against viral infections. RIG-I recognizes viral RNA and activates the type I IFN-dependent antiviral innate-immune response (Wang et al., 2013). RIG-I is reported to act independently of the TLRs (Foy et al., 2005), and RIG-I signaling culminates in the induction of IFN- $\alpha / \beta$, which inhibits viral replication without killing infected cells (Benedict and Ware, 2005). It has been demonstrated that the activation of RIG-I signaling could inhibit a number of viruses, including the hepatitis C virus (Saito et al., 2008; Wang et al., 2013), ebolavirus (Spiropoulou et al., 2009), and influenza virus (Ranjan et al., 2010). Recent studies (Solis et al., 2011; Berg et al., 2012) indicated that RIG-I is involved in control of HIV replication, as RIG-I could recognize the secondary-structured RNA of HIV, leading to the activation of innate-immune responses (Berg et al., 2012). However, RIG-I-dependent antiviral signaling is inhibited by HIV infection (Solis et al., 2011). Thus, to activate RIG-I by its ligand represents a promising approach for the treatment of HIV infection. The crucial finding we report in this study is that RIG-I senses HIV-1 infection and induces a type-I IFN response in human MDMs.

We have previously shown that HIV-1 infection can decrease the expression of interferon regulatory factor (IRF)-1, IRF-7 gene and STAT1 activation in a human MDM culture system (Huang et al., 2009). In this study, we examined the pathways in human MDM that could induce a type I IFN response. Cherner et al. (2002) showed that HIV-1 replication in the CNS is associated with neurocognitive impairment. To the best our knowledge, this is the first time that it has been demonstrated that both cytosolic RNA sensors RIG-I and MDA5 were significantly upregulated in the brains of HIVE patients. Furthermore, RIG-I and MDA5 expression levels were correlated with STAT1 expression and the activation of type I IFN response. Additionally, HIV-1 infection induced the expression of both RIG-I and MDA5 in MDMs and this elevation correlated with the type I IFN response. HIV-1-infected MDMs induced the type I IFN response in a RIG-I-dependent manner. As our data show, RIG-I silencing can inhibit activation of STAT1 and enhance HIV-1 replication in HIV1 -infected MDMs. The evidence provided here strongly suggests that RIG-I signaling components participate in the innate immune response in HIV-infected MDMs.

Taken together, our study provides compelling experimental evidence that RIG-I activation in HIVE tissue can significantly suppresses HIV infection and replication in MDMs through multiple antiviral mechanisms at cellular and molecular levels. Although additional mechanism(s) might also be involved, the induction of type I IFNs and IFN-inducible antiviral factors should account for much of the RIG-I activation-mediated anti-HIV activity. Determining the molecular mechanism of HIV-1 detection and immune response in human MDMs may help us to further understand the neuropathogenesis of HAND, which may provide a novel method in the treatment and diagnosis of HAND.

\section{Conflicts of Interest}

The authors declare no conflict of interest.

\section{ACKNOWLEDGMENTS}

Author contributions: M.W., Y.H., G.Q., and J.C.Z. designed research; M.W., Y.H., J.H., 
G.Q., and J.C.Z. performed research; M.W., Y.H., J.H., G.Q., and J.C.Z. contributed unpublished reagents/analytic tools; M.W., Y.H., G.Q., and J.C.Z. analyzed data; and M.W., Y.H., and G.Q. wrote the paper. This work was supported in part by the following research grants: National Institutes of Health (\#R01 NS 41858-01) and "973" program of China (\#2004CB518804). We sincerely thank Dr. Changhai Tian, Dr. Hui Peng, Dr. Yongxiang Wang, and Ling Ye, who provided support for this work. We thank Dr. Yuri Persidsky and Dr. Anuja Ghorpade, who assisted with the brain specimen collection.

\section{REFERENCES}

Benedict CA and Ware CF (2005). RIGing a virus trap. Nat. Med.11: 929-930.

Berg RK, Melchjorsen J, Rintahaka J, Diget E, et al. (2012). Genomic HIV RNA induces innate immune responses through RIG-I-dependent sensing of secondary-structured RNA. PloS One 7: e29291.

Borrow P, Shattock RJ and Vyakarnam A (2010). Innate immunity against HIV: a priority target for HIV prevention research. Retrovirology 7: 84

Brown MG, McAlpine SM, Huang YY, Haidl ID, et al. (2012). RNA sensors enable human mast cell anti-viral chemokine production and IFN-mediated protection in response to antibody-enhanced dengue virus infection. PLoS One 7: e34055.

Burkala EJ, He J, West JT, Wood C, et al. (2005). Compartmentalization of HIV-1 in the central nervous system: role of the choroid plexus. AIDS 19: 675-684.

Carter CA and Ehrlich LS (2008). Cell biology of HIV-1 infection of macrophages. Annu. Rev. Microbiol. 62: $425-443$.

Cherner M, Masliah E, Ellis RJ, Marcotte TD, et al. (2002). Neurocognitive dysfunction predicts postmortem findings of HIV encephalitis. Neurology 59: 1563-1567.

Foy E, Li K, Sumpter R Jr, Loo YM, et al. (2005). Control of antiviral defenses through hepatitis C virus disruption of retinoic acid-inducible gene-I signaling. Proc. Nat. Acad. Sci. U.S.A. 102: 2986-2991.

Fujita T, Onoguchi K, Onomoto K, Hirai R, et al. (2007). Triggering antiviral response by RIG-I-related RNA helicases. Biochimie 89: 754-760.

Gendelman HE, Orenstein JM, Martin MA, Ferrua C, et al. (1988). Efficient isolation and propagation of human immunodeficiency virus on recombinant colony-stimulating factor 1-treated monocytes. J. Exp. Med. 167: 1428-1441.

Huang Y, Walstrom A, Zhang L, Zhao Y, et al. (2009). Type I interferons and interferon regulatory factors regulate TNF-related apoptosis-inducing ligand (TRAIL) in HIV-1-infected macrophages. PLoS One 4: e5397.

Huang Y, Zhao L, Jia B, Wu L, et al. (2011). Glutaminase dysregulation in HIV-1-infected human microglia mediates neurotoxicity: relevant to HIV-1-associated neurocognitive disorders. J. Neurosci. 31: 15195-15204.

Katze MG, He Y and Gale M Jr. (2002). Viruses and interferon: a fight for supremacy. Nat. Rev. Immunol. 2: 675-687.

Kawai T and Akira S (2008). Toll-like receptor and RIG-l-like receptor signaling. Ann. N.Y. Acad. Sci. 1143: 1-20.

Koenig S, Gendelman HE, Orenstein JM, Dal Canto MC, et al. (1986). Detection of AIDS virus in macrophages in brain tissue from AIDS patients with encephalopathy. Science 233: 1089-1093.

Marq JB, Hausmann S, Veillard N, Kolakofsky D, et al. (2011). Short double-stranded RNAs with an overhanging 5' pppnucleotide, as found in arenavirus genomes, act as RIG-I decoys. J. Biol. Chem. 286: 6108-6116.

Perno CF, Svicher V, Schols D, Pollicita M, et al. (2006). Therapeutic strategies towards HIV-1 infection in macrophages. Antiviral Res. 71: 293-300.

Raivich G and Banati R (2004). Brain microglia and blood-derived macrophages: molecular profiles and functional roles in multiple sclerosis and animal models of autoimmune demyelinating disease. Brain Res. Brain Res. Rev. 46: $261-281$.

Ranjan P, Jayashankar L, Deyde V, Zeng H, et al. (2010). 5'PPP-RNA induced RIG-I activation inhibits drug-resistant avian H5N1 as well as 1918 and 2009 pandemic influenza virus replication. Virol. J. 7: 102.

Rehwinkel J, Tan CP, Goubau D, Schulz O, et al. (2010). RIG-I detects viral genomic RNA during negative-strand RNA virus infection. Cell 140: 397-408.

Saito T, Owen DM, Jiang F, Marcotrigiano J, et al. (2008). Innate immunity induced by composition-dependent RIG-I recognition of hepatitis C virus RNA. Nature 454: 523-527.

Solis M, Nakhaei P, Jalalirad M, Lacoste J, et al. (2011). RIG-I-mediated antiviral signaling is inhibited in HIV-1 infection by a protease-mediated sequestration of RIG-I. J. Virol. 85: 1224-1236.

Spiropoulou CF, Ranjan P, Pearce MB, Sealy TK, et al. (2009). RIG-I activation inhibits ebolavirus replication. Virology 392: 11-15.

Takeuchi O and Akira S (2010). Pattern recognition receptors and inflammation. Cell 140: 805-820.

Verani A, Gras G and Pancino G. (2005). Macrophages and HIV-1: dangerous liaisons. Mol. Immunol. 42: $195-212$.

Wang Y, Wang X, Li J, Zhou Y, et al. (2013). RIG-I activation inhibits HIV replication in macrophages. J. Leukoc. Biol. 94: $337-341$. 
Wang Y, Ye L, Wang X, Li J, et al. (2013). Retinoic acid inducible gene-I (RIG-I) signaling of hepatic stellate cells inhibits hepatitis C virus replication in hepatocytes. Innate Immun. 19: 193-202.

Witwer KW, Gama L, Li M, Bartizal CM, et al. (2009). Coordinated regulation of SIV replication and immune responses in the CNS. PLoS One 4: e8129.

Zhang H, Hoffmann F, He J, He X, et al. (2005). Evolution of subtype C HIV-1 Env in a slowly progressing Zambian infant. Retrovirology 2: 67.

Zhang H, Hoffmann F, He J, He X, et al. (2006). Characterization of HIV-1 subtype C envelope glycoproteins from perinatally infected children with different courses of disease. Retrovirology 3: 73.

Zhao L, Huang Y, Tian C, Taylor L, et al. (2012). Interferon-alpha regulates glutaminase 1 promoter through STAT1 phosphorylation: relevance to HIV-1 associated neurocognitive disorders. PLoS One 7: e32995. 